\title{
Interacting spin-1 bosons in a two-dimensional optical lattice
}

\author{
L. de Forges de Parny ${ }^{1,2}$, F. Hébert ${ }^{1}$, V.G. Rousseau ${ }^{3}$, and G.G. Batrouni ${ }^{1,4,5}$ \\ ${ }^{1}$ INLN, Université de Nice-Sophia Antipolis, CNRS; 1361 route des Lucioles, 06560 Valbonne, France, \\ ${ }^{2}$ Institute of Theoretical Physics, Ecole Polytechnique Fédérale de Lausanne (EPFL), CH-1015 Lausanne, Switzerland, \\ ${ }^{3}$ Department of Physics and Astronomy, Louisiana State University, Baton Rouge, Louisiana 70803, USA, \\ ${ }^{4}$ Institut Universitaire de France, 103 bd Saint-Michel, 75005 Paris, France, and \\ ${ }^{5}$ Centre for Quantum Technologies, National University of Singapore; 2 Science Drive 3 Singapore 117542
}

\begin{abstract}
We study, using quantum Monte Carlo (QMC) simulations, the ground state properties of spin-1 bosons trapped in a square optical lattice. The phase diagram is characterized by the mobility of the particles (Mott insulating or superfluid phase) and by their magnetic properties. For ferromagnetic on-site interactions, the whole phase diagram is ferromagnetic and the Mott insulators-superfluid phase transitions are second order. For antiferromagnetic on-site interactions, spin nematic order is found in the odd Mott lobes and in the superfluid phase. Furthermore, the superfluid-insulator phase transition is first or second order depending on whether the density in the Mott is even or odd. Inside the even Mott lobes, we observe a singlet-to-nematic transition for certain values of the interactions. This transition appears to be first order.
\end{abstract}

PACS numbers: $\quad$ 05.30.Jp, 03.75.Hh, 67.40.Kh, 75.10.Jm 03.75.Mn

\section{INTRODUCTION}

Ultracold atoms in optical lattices have been used in the recent years to explore the physics of well known quantum statistical discrete models [1], such as the Bose [2] and Fermi 3, 44 single-band Hubbard models. Purely optical traps [5] allow the trapping of alkali atoms, such as ${ }^{23} \mathrm{Na},{ }^{30} \mathrm{~K}$, and ${ }^{87} \mathrm{Rb}$, in the $F=1$ hyperfine state without freezing $F_{z}$. The presence of these spin degrees of freedom allows the study of multi-band condensed matter Hamiltonians and the interplay between magnetism and superfluidity [6, 7].

Such systems are governed by extended Hubbard Hamiltonians, with spin-dependent terms [8, 9], able to describe the spinful nature of the particles. The on-site spin-spin interaction can be tuned by using optical Feshbach resonance [10] and the nature of the spin-spin interaction can be either ferromagnetic $\left({ }^{87} \mathrm{Rb}\right)$ or antiferromagnetic $\left({ }^{23} \mathrm{Na}\right)$ depending on the relative magnitudes of the scattering lengths in the singlet and quintuplet channels [11]. In the strong coupling limit and with integer filling, the spinor Bose-Hubbard model can be mapped onto the Heisenberg model with biquadratic interactions which exhibits singlet, dimerized or nematic phases 12 14.

Several approaches have been used to study the spin-1 bosonic model: mean field methods [15, 16], variational Monte Carlo 17], analytical 12, 18], strong coupling expansion [19], Density Matrix Renormalization Group (DMRG) 20, 21], and quantum Monte Carlo simulations in 1D [22, 23]. The overall picture emerging at zero temperature is that, as expected, the system adopts Mottinsulating (MI) phases, when the filling is commensurate with the lattice size and for large enough repulsion between particles, and a superfluid phase (SF) otherwise. The richness of these systems comes from the magnetic behavior of these phases that changes dramatically with the sign of the spin-dependent interaction parameter $U_{2}$.
For negative spin-dependent interaction $U_{2}<0$ (see below) corresponding to the ferromagnetic case, a 1D QMC study 23] shows that all Mott lobes shrink, and eventually disappear, as one increases $\left|U_{2}\right|$. The whole phase diagram is ferromagnetic and the same is expected in larger dimension. This was confirmed by a recent theorem that shows that the ground state of such a system is always ferromagnetic [18].

The positive $U_{2}$ antiferromagnetic case exhibits a richer phase diagram. For even densities, $\rho$, the Mott lobes are expected to have spin singlets on every site but a first order transition to a nematic state is predicted for $U_{2} / U_{0}<0.025$ in two dimensions $\left.12,24,25\right]$. For odd $\rho$, a nematic order is also expected in two dimensions, depending on the interaction $U_{2}$ [12] (in one dimension, this nematic phase is changed into a dimerized phase [20, 23]). The superfluid phase is expected to be polarized: superfluidity is carried either by the $F_{z}=0$ component or by the $F_{z}= \pm 1$ components 15 . The theorem previously mentioned [18] proves that the global magnetization is zero in the whole phase diagram in the subspace of zero total spin along the $z$ axis $\left(F_{\text {tot }, z}=0\right)$. This is compatible with the nematic or dimerized phases observed in other studies.

As for the nature of the MI-SF phase transitions, it was predicted to be affected by the spin-spin interactions [15, 16]. For $\rho=2$ it is argued to be first-order for small positive $U_{2}$ (and second order in all other cases) whereas the transition for $\rho=1$ should always be second order. The one-dimensional QMC investigation [23] presented evidence of singlet and dimerized phases but found only second order transitions, which is expected in one dimension. Recent 1D and 2D QMC studies [26, 27] of a similar system with only two species showed a first order transition for even lobes occurring in two dimensions, as predicted by previous mean-field studies [28, 29], but not in the one-dimensional case.

The purpose of this paper is to extend the QMC study 
of the spin-1 system to the two-dimensional square lattice at zero temperature. The paper is organized as follows: in Sec. II, we will introduce the model and QMC techniques used to study it. Secs. III and IV will be devoted to the presentation of the results obtained for the $U_{2}<0$ and $U_{2}>0$ cases, respectively. In Sec. V, we will summarize these results and give some final remarks.

\section{SPIN-1 MODEL}

We consider a system of bosonic atoms in the hyperfine state $F=1$ characterized by the magnetic quantum number $F_{z}=0, \pm 1$. When these atoms are loaded in an optical lattice, the system is governed by the BoseHubbard Hamiltonian [8, 24]:

$$
\begin{aligned}
\mathcal{H}= & -t \sum_{\sigma,\left\langle\mathbf{r}, \mathbf{r}^{\prime}\right\rangle}\left(a_{\sigma \mathbf{r}}^{\dagger} a_{\sigma \mathbf{r}^{\prime}}+\text { h.c. }\right)+\frac{U_{0}}{2} \sum_{\mathbf{r}} \hat{n}_{\mathbf{r}}\left(\hat{n}_{\mathbf{r}}-1\right) \\
& +\frac{U_{2}}{2} \sum_{\mathbf{r}}\left(\mathbf{F}_{\mathbf{r}}^{2}-2 \hat{n}_{\mathbf{r}}\right)
\end{aligned}
$$

where operator $a_{\sigma \mathbf{r}}\left(a_{\sigma \mathbf{r}}^{\dagger}\right)$ annihilates (creates) a boson of spin $\sigma=-1,0,1$ (also denoted $-, 0,+$ when needed) on site $\mathbf{r}$ of a periodic square lattice of size $L \times L$.

The first term in the Hamiltonian is the kinetic term which allows particles to hop between neighboring sites $\left\langle\mathbf{r}, \mathbf{r}^{\prime}\right\rangle$. The hopping parameter $t=1$ sets the energy scale and the number operator $\hat{n}_{\mathbf{r}} \equiv \sum_{\sigma} \hat{n}_{\sigma \mathbf{r}}=$ $\sum_{\sigma} a_{\sigma \mathbf{r}}^{\dagger} a_{\sigma \mathbf{r}}$ counts the total number of bosons on site $\mathbf{r}$. $N_{\sigma}=\sum_{\mathbf{r}} n_{\sigma \mathbf{r}}$ will denote the total number of $\sigma$ bosons, $\rho_{\sigma}=N_{\sigma} / L^{2}$ the corresponding density, and $\rho$ the total density. The operator $\mathbf{F}_{\mathbf{r}}=\left(F_{x, \mathbf{r}}, F_{y, \mathbf{r}}, F_{z, \mathbf{r}}\right)$ is the spin operator where $F_{\alpha, \mathbf{r}}=\sum_{\sigma, \sigma^{\prime}} a_{\sigma \mathbf{r}}^{\dagger} J_{\alpha, \sigma \sigma^{\prime}} a_{\sigma^{\prime} \mathbf{r}}, \alpha=x, y, z$ and the $J_{\alpha, \sigma \sigma^{\prime}}$ are standard spin-1 matrices. Once developed, the $\mathbf{F}_{\mathbf{r}}^{2}$ term exhibits contact interaction terms and conversion terms between the species. The parameters $U_{0}$ and $U_{2}$ are the on-site spin-independent and spindependent interaction terms. The nature of the bosons will give the sign of $U_{2}$ and consequently the nature of the on-site spin-spin interaction whereas the spin independent part is always repulsive, $U_{0}>0$. The $U_{2}<0$ case (e.g. $\left.{ }^{87} \mathrm{Rb}\right)$ will maximize the local magnetic moment $F^{2}(0) \equiv \sum_{\mathbf{r}}\left\langle\mathbf{F}_{\mathbf{r}}^{2}\right\rangle / L^{2}$. Therefore, this is referred to as the "ferromagnetic" case whereas the $U_{2}>0$ case (e.g. ${ }^{23} \mathrm{Na}$ ) which favors minimum on-site spins is referred to as "antiferromagnetic".

To simulate this system, we used the Stochastic Green Function algorithm (SGF) 30] with directed updates [31], an exact Quantum Monte Carlo technique that allows canonical or grand canonical simulations of the system as well as measurements of many-particle Green functions. In particular, this algorithm can simulate efficiently the spin-flip term $a_{0 \mathbf{r}}^{\dagger} a_{0 \mathbf{r}}^{\dagger} a_{-\mathbf{r}} a_{+\mathbf{r}}+$ h.c. which appears in the development of the $\mathbf{F}_{\mathbf{r}}^{2}$ term. In this work we used mostly the canonical formulation where the total density $\rho$ is conserved whereas the individual densities $\rho_{\sigma}$ fluctuate. The QMC algorithm also conserves the total spin along $z, F_{\text {tot, } z}=N_{+}-N_{-}$, which adds a constraint to the canonical one. The value of $F_{\text {tot, } z}$ in a given canonical simulation is then fixed by the choice of the initial numbers of particles. Due to this constraint, the magnetic physical quantities, involving $F_{\alpha, \mathbf{r}}$ operators, are identical for the $x$ and $y$ axes but may be different for the $z$ axis. The initial symmetry, where all three axes should behave identically, is broken in our simulations. Using this algorithm we were able to simulate the system reliably for clusters going up to $L \times L=12 \times 12$ and $\beta=2 L / t$. We generally compared the results for sizes $L=8,10$, and 12 to check that there was no appreciable difference in the behaviour of the system within the limit of the precision we could achieve.

At zero temperature, we relate the density to the chemical potential $\mu$ using

$$
\mu(N)=E(N+1)-E(N),
$$

where $N$ is the total number of bosons, and $E=\langle\mathcal{H}\rangle$ is the average energy, equal to the free energy in the ground state.

The superfluid density is given by 32]

$$
\rho_{s}=\frac{\left\langle W^{2}\right\rangle}{4 t \beta},
$$

where the total winding number $W=W_{-}+W_{0}+W_{+}$is a topological quantity, and $\beta=1 / k_{B} T$. The analysis of the magnetic structure requires the equal-time spin-spin correlation functions

$$
F_{\alpha \alpha}(\mathbf{R})=\frac{1}{L^{2}} \sum_{\mathbf{r}}\left\langle F_{\alpha, \mathbf{r}} F_{\alpha, \mathbf{r}+\mathbf{R}}\right\rangle .
$$

The global magnetization is given by

$$
F_{\text {tot }}^{2}=L^{2} \sum_{\alpha, \mathbf{R}} F_{\alpha \alpha}(\mathbf{R})
$$

and the magnetic structure factor by

$$
S(\mathbf{k})=\frac{1}{L^{2}} \sum_{\alpha, \mathbf{R}} e^{i \mathbf{k} \cdot \mathbf{R}} F_{\alpha \alpha}(\mathbf{R})
$$

where $\mathbf{k}=\left(k_{x}, k_{y}\right)$ and $k_{x, y}$ are integer multiples of $2 \pi / L$. Other important quantities are the diagonal components of the nematic order parameter traceless tensor $\mathbf{Q}$ given by [24]

$$
Q_{\alpha \alpha}=\left\langle F_{\alpha \alpha}(0)-\frac{1}{3} F^{2}(0)\right\rangle .
$$

These are the indicators of the on-site spin isotropy if $Q_{\alpha \alpha}=0$ for all $\alpha$, and of the spin anisotropy characteristic of the nematic order if $Q_{\alpha \alpha} \neq 0$ and if, at the same time, no magnetic order is present. As $Q_{x x}=Q_{y y}$ in our simulations, then $2 Q_{x x}+Q_{z z}=0$ and it is sufficient to calculate $Q_{z z}$. Since nematic order can develop 
along any axis and as there is not always an ergodicity breaking in $\mathrm{MC}$ simulations on finite clusters, $Q_{z z}$ can change sign and average to zero even if it is non-zero in each individual measurements. To avoid such an effect, we use the absolute value of the sums of spin operators in $Q_{z z}^{\prime}=\left\langle\left|F_{z z}(0)-\frac{1}{3} F^{2}(0)\right|\right\rangle$.

We also calculate the Green functions

$$
G_{\sigma}(\mathbf{R})=\frac{1}{2 L^{2}} \sum_{\mathbf{r}}\left\langle a_{\sigma \mathbf{r}+\mathbf{R}}^{\dagger} a_{\sigma \mathbf{r}}+a_{\sigma \mathbf{r}}^{\dagger} a_{\sigma \mathbf{r}+\mathbf{R}}\right\rangle,
$$

which measure the phase coherence of particles. The anticorrelated motions of particles, which govern the dynamics inside Mott lobes, as the particles of different types exchange their positions are described by the twoparticle anticorrelated Green functions

$$
G_{a}\left(\sigma \sigma^{\prime}, \mathbf{R}\right)=\frac{1}{2 L^{2}} \sum_{\mathbf{r}}\left\langle\hat{a}_{\sigma \mathbf{r}+\mathbf{R}}^{\dagger} \hat{a}_{\sigma^{\prime} \mathbf{r}+\mathbf{R}} \hat{a}_{\sigma^{\prime} \mathbf{r}}^{\dagger} \hat{a}_{\sigma \mathbf{r}}+\text { h.c. }\right\rangle \text {. }
$$

If perfect phase coherence is established by means of particle exchange, $G_{a}\left(\sigma \sigma^{\prime}, \mathbf{R}\right)$ reaches its limiting upper value of $\rho_{\sigma} \rho_{\sigma^{\prime}}$ at long distances $\mathbf{R}$. Due to its definition, $G_{a}\left(\sigma \sigma^{\prime}, \mathbf{R}\right)=G_{\sigma}(\mathbf{R}) G_{\sigma^{\prime}}(\mathbf{R})$ if there is no correlation between the movements of particles of spin $\sigma$ and $\sigma^{\prime}$.

\section{FERROMAGNETIC CASE: $U_{2}<0$}

We start with the ferromagnetic case, $U_{2}<0$. Several approaches have been used to study this case. A recent theorem by Katsura and Tasaki, independent of the lattice dimension, shows that the ground state universally exhibits saturated ferromagnetism both in superfluid and Mott insulator phases [18]. Mean field analyses predicted the same magnetic behavior and continuous MI-SF phase transitions [15, 16]. The 1D investigation [23], by means of QMC simulations, showed that the magnetic local moment on a site with $n$ bosons is maximized $F^{2}(0)=n(n+1)$ and that the whole phase diagram is ferromagnetic, in agreement with the above theorem. In the $2 \mathrm{D}$ system, studied with variational Monte Carlo at $\rho=1$ [17], Mott and superfluid phases were also found to be ferromagnetic. In the first subsection, we calculate the $2 \mathrm{D}$ phase diagram in the $\left(\mu / U_{0}, t / U_{0}\right)$ plane at a fixed ratio $U_{2} / U_{0}=-0.1$ and we discuss the phase transitions in the second subsection.

\section{A. Phase diagram}

In the no-hopping limit, $t / U_{0} \rightarrow 0$, the system is in a MI phase with $n$ bosons per site with energy per site $\varepsilon(n)=n(n-1)\left(U_{0}+U_{2}\right) / 2$ when $\mu$ satisfies the condition $(n-1)\left(U_{0}+U_{2}\right)<\mu<n\left(U_{0}+U_{2}\right)$. Therefore, the bases of both the odd and even Mott lobes, given by $\Delta \mu / U_{0}=1-\left|U_{2}\right| / U_{0}$, shrink with increasing $\left|U_{2}\right|$ and disappear completely when $\left|U_{2}\right|=U_{0}$. The on-site energy is minimized by maximizing the quantum number
$F$ corresponding to $F^{2}(0)=F(F+1)$, that is by adopting any of the $F=1$ triplet states for $\rho=1$ and any of the $F=2$ quintuplet states for $\rho=2$. Thus, in the $t / U_{0} \rightarrow 0$ limit, the Mott phases are highly degenerate. As $t / U_{0}$ increases, the MI regions are reduced and eventually disappear, giving the familiar Mott lobes (Fig. 10. Outside the MI region the system is superfluid. Note that the shape of the lobes is very different from the $1 \mathrm{D}$ case 23] where the lobes end in cusps. The MF results obtained with the technique used in [15] are in very good agreement with QMC results at large interactions, as expected. The tip of the $\rho=2$ is also well reproduced by the MF approximation whereas there is a quantitative difference between QMC and MF for $\rho=1$.

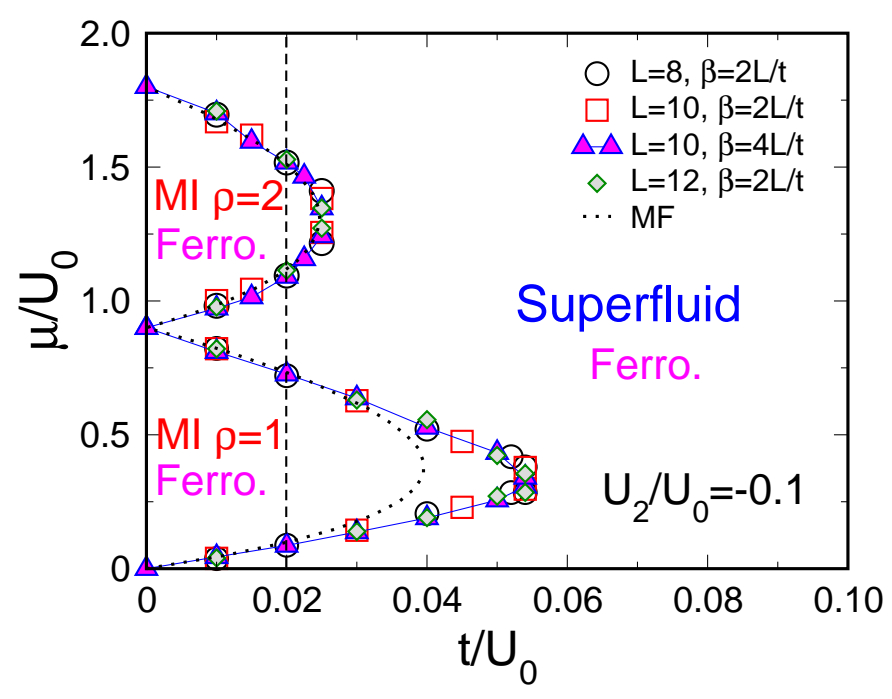

FIG. 1: (Color online) $F_{\text {tot, } z}=0$ phase diagram at $\beta=2 L / t$ or $\beta=4 L / t$ for $U_{2} / U_{0}=-0.1$ and several linear system sizes $L$. All phases are ferromagnetic and all transitions are continuous. The dashed vertical line indicates where the cut in Fig. 8 was taken. The dotted line corresponds to the MF phase diagram obtained with the technique used in [15].

In the Mott insulator phases, the superfluid density is zero and the densities are $\rho_{0}=2 \rho_{+}=2 \rho_{-}=\rho / 2$. The degenerate states of the MI phases in the $t / U_{0} \rightarrow 0$ limit are coupled by second-order contributions from the hopping term which exchange the position of different particles. The degeneracy is, then, lifted by establishing a phase coherence of such exchanges [27, 33, 34]. Figure[2 shows single-particle and anticorrelated Green functions in the MI phases for $\rho=1$ and $\rho=2$. As expected in the MI phase, we see that the individual Green functions $G_{\sigma}(\mathbf{R})$ decay exponentially to zero with distance R. Figure 2 shows that the $G_{a}\left(\sigma \sigma^{\prime}, \mathbf{R}\right)$ quickly saturate to a constant value at large separations, indicating coherent exchange moves in the MI phase for both $\rho=1$ and $\rho=2$. Furthermore, the plateau reached by this function takes on its maximum possible value at large distances, $\rho_{\sigma} \rho_{\sigma^{\prime}}$, showing perfect phase coherence. The equalities $G_{-}(\mathbf{R})=G_{+}(\mathbf{R})$ and $G_{a}(+0, \mathbf{R})=G_{a}(-0, \mathbf{R})$ are due to the $F_{\text {tot, } z}=0$ constraint. As $\rho_{0}>\rho_{ \pm}$, the Green func- 
tions involving spin-0 particles $\left(G_{0}(\mathbf{R})\right.$ and $\left.G_{a}( \pm 0, \mathbf{R})\right)$ are larger than Green functions involving only \pm 1 particles, which will also be true in the superfluid case.
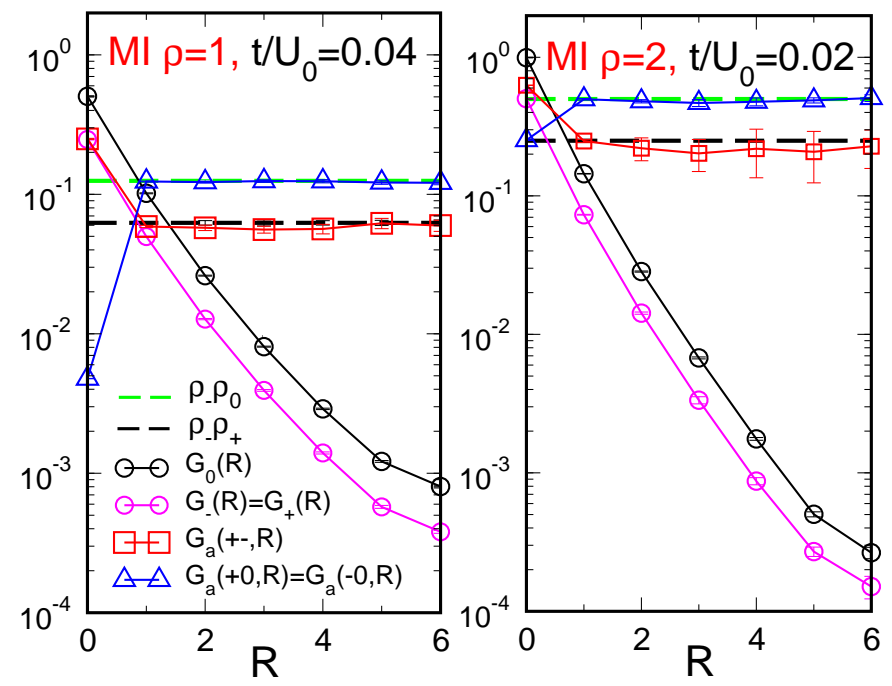

FIG. 2: (Color online) The single-particle and the anticorrelated Green functions for $U_{2} / U_{0}=-0.1, L=12$ and $\beta=2 L / t$ as a function of distance $R$ in the Mott insulator phases with $\rho=1$ and $\rho=2$. Individual movements of particles are strongly suppressed, as shown by the exponential decay of $G_{0}$ and $G_{-}=G_{+}$, whereas perfect anticorrelated movements of particles remain, which is shown by the plateau in $G_{a}(+-) \rightarrow \rho_{-} \rho_{+}$and $G_{a}(+0)=G_{a}(-0) \rightarrow \rho_{ \pm} \rho_{0}$ when $R \rightarrow L / 2$.

In the superfluid phase, we observe the same density distribution $\rho_{0}=2 \rho_{+}=2 \rho_{-}=\rho / 2$. Figure 3 shows phase coherence both for exchange moves and for individual movements of particles. The saturation of the single-particle Green functions $G_{\sigma}(\mathbf{R})$ to a constant value at large separations is the signature of the existence of a Bose-Einstein condensate as the condensed fraction is given by $\rho(\mathbf{k}=0)=\sum_{\sigma} \rho_{\sigma}(\mathbf{k}=0)$, where the $\rho_{\sigma}(\mathbf{k})$ are Fourier transforms of single-particle Green functions. For both densities, $\rho=1$ and $\rho=2$, the three species remain correlated, of course, since the system is still in a strongly interacting regime $\left(t / U_{0}=0.10\right)$. This correlation can be inferred from the fact that $G_{a}(+-, \mathbf{R})>G_{+}(\mathbf{R}) G_{-}(\mathbf{R})$ and $G_{a}( \pm 0, \mathbf{R})>G_{ \pm}(\mathbf{R}) G_{0}(\mathbf{R})$ at large separations. This means that, while particles can move independently, exchanges of different particles are still present. This is typical of a strongly correlated superfluid where different kinds of phase coherence can be observed: phase coherence of the individual particles, but also, at the same time, phase coherence of exchange moves of particles.

We now focus on the magnetic properties of MI and SF phases for $U_{2} / U_{0}=-0.1$. The ground state exhibits saturated ferromagnetism in the whole phase diagram, as predicted by Katsura and Tasaki's theorem [18] and previously observed in 17, 23]. In both MI and SF phases with integer density $\rho=1,2$ we measure the largest pos-
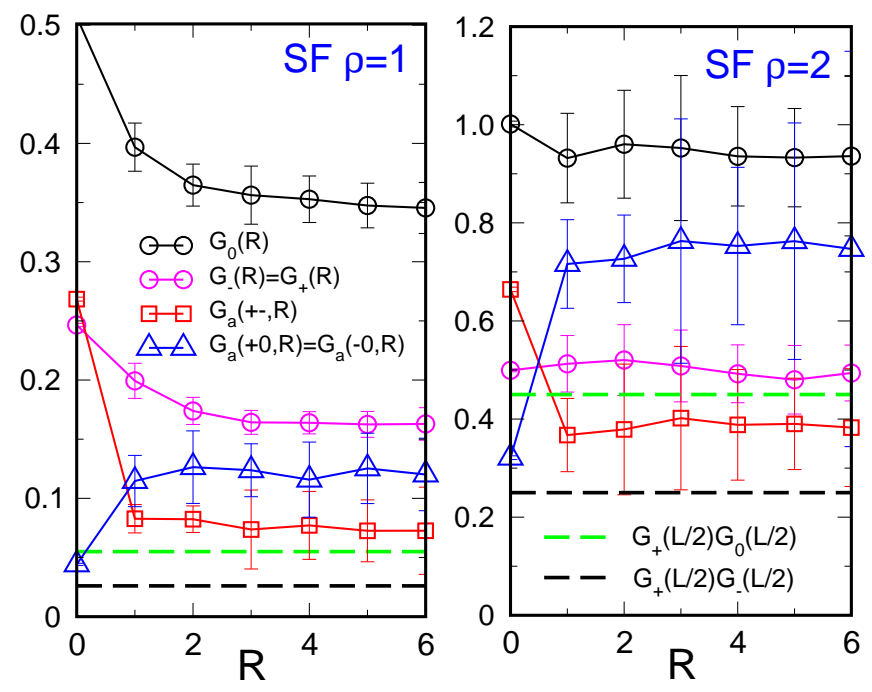

FIG. 3: (Color online) The single-particle and the anticorrelated Green functions for $U_{2} / U_{0}=-0.1, L=12, \beta=2 L / t$ with $t / U_{0}=0.10$ as a function of distance $R$ in the superfluid phase with $\rho=1$ and $\rho=2$. The long-range order of the Green functions indicates both individual and anticorrelated movements of particles.

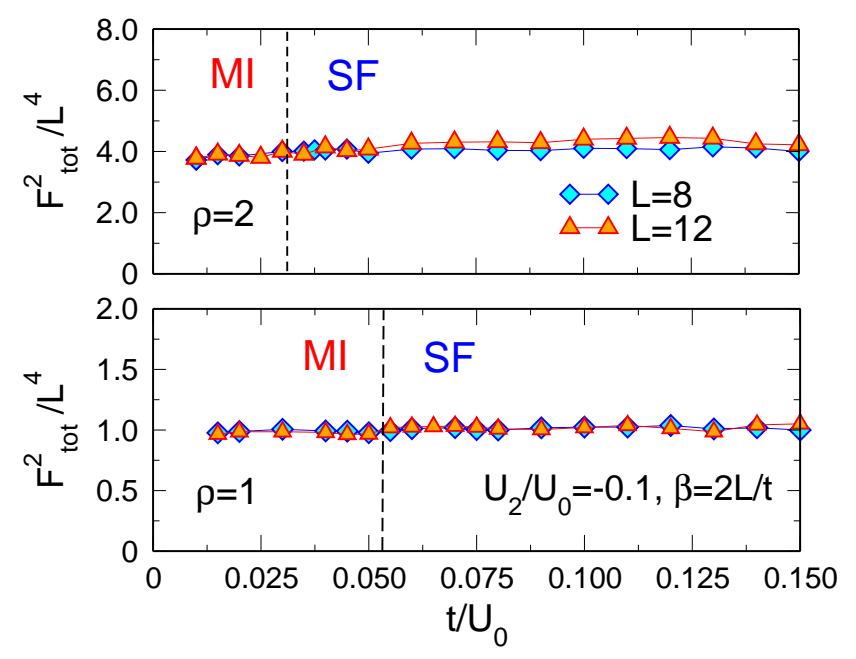

FIG. 4: (Color online) The normalized total magnetization $F_{\text {tot }}^{2}$ versus $t / U_{0}$ at fixed total density $\rho=1,2$. The dashed vertical lines indicate the critical values $t_{c} / U_{0}$ where the MISF phase transitions occur.

sible spin on-site, given by $F^{2}(0)=\rho(\rho+1)$. Furthermore, the ground state has the maximum possible total spin $F_{\text {tot }}^{2}=N(N+1)$, thus $F_{\text {tot }}^{2} / L^{4}=\rho\left(\rho+1 / L^{2}\right) \simeq \rho^{2}$ (Fig. 4) in the whole phase diagram, in the superfluid phase as well as in the Mott phase. Figure 5 shows that the ground state energy $E\left(F_{\mathrm{tot}, z}\right)$ is independent of the $F_{\text {tot }, z}$ chosen in the simulation, in the MI or in the SF phase with $\rho=1$ or 2 . As mentionned earlier, $F_{\mathrm{tot}, z}$ is imposed by the initial values of $N_{+}$and $N_{-}$used in a given simulation. The ground states are then $(2 N+1)$ - 
fold degenerate, as expected if $F_{\text {tot }}^{2}=N(N+1)$. This also shows that the constant $F_{\text {tot, } z}$ we impose just select one of these $2 N+1$ states and does not alter the physics.

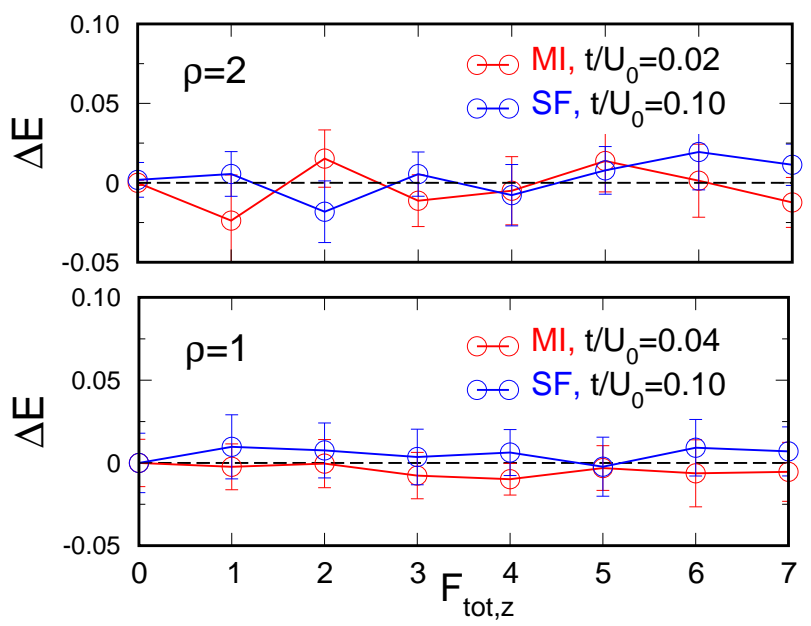

FIG. 5: (Color online) The energy difference $\Delta E=$ $E\left(F_{\text {tot }, z}\right)-E\left(F_{\text {tot }, z}=0\right)$ versus $F_{\text {tot }, z}$ in the MI and SF phases for $\rho=1,2$ with $U_{2} / U_{0}=-0.1, L=4$ and $\beta=6 L / t . \Delta E$ is zero, in the error bars limit, indicating that the ground states are $(2 N+1)$-fold degenerate.

Figure [6 shows $F_{\text {tot }}^{2} / L^{4}, F^{2}(0)$, and the superfluid density $\rho_{s}$ versus the density $\rho$. It shows that $F_{\text {tot }}^{2}=$ $N(N+1)$ and that the magnetic properties are not influenced by the superfluid or solid nature of the underlying phases. The linear step by step evolution of $F^{2}(0)$ is simply explained by the strong on-site repulsion $U_{0}$. For a density $\rho$ we define $\rho_{i}$, the integer part of the density (for $2 \leq \rho<3, \rho_{i}=2$ ). To minimize the repulsion cost, a system with density $\rho$ will have the minimal number of sites occupied by $\rho_{i}+1$ particles, that is $\left(\rho-\rho_{i}\right) L^{2}$. These sites contribute $\left(\rho_{i}+1\right)\left(\rho_{i}+2\right)$ to the local moment and the remaining $\left(\rho_{i}+1-\rho\right) L^{2}$ sites occupied by $\rho_{i}$ particles contribute $\rho_{i}\left(\rho_{i}+1\right)$. Averaging these contributions yields the observed dependence which is $F^{2}(0)=\left(\rho_{i}+1\right)\left(2 \rho-\rho_{i}\right)$.

\section{B. Phase transitions}

Figure 7 shows the evolution of the superfluid density $\rho_{s}$ at the MI-SF transition at fixed total density while varying $t / U_{0}$, in other words the transition at the tip of the Mott lobe. All quantum phase transitions appear to be continuous and there are no signs of possible discontinuities in the superfluid density at the transition between the MI and SF phases. The finite size effects are small and the transitions appear continuous, which means that they should be in the universality class of the three-dimensional XY model, which is expected from

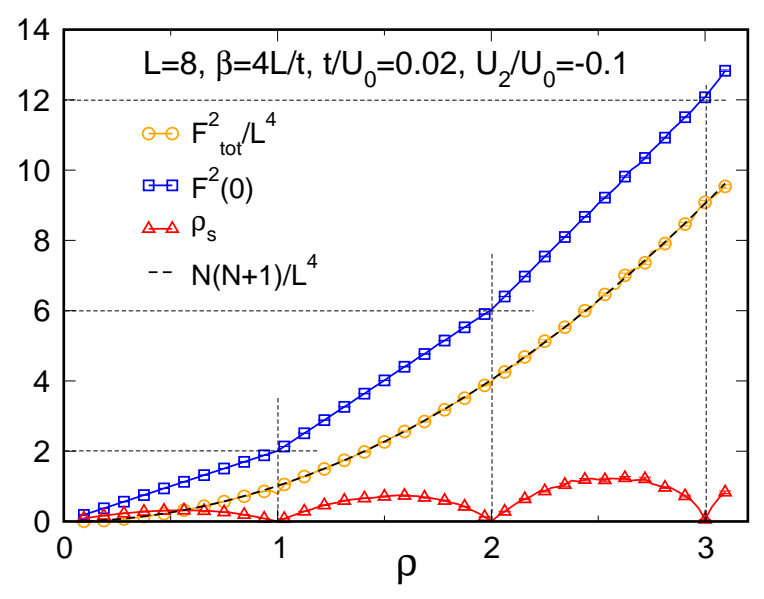

FIG. 6: (Color online) Normalized total magnetization $F_{\text {tot }}^{2} / L^{4}$, local magnetic moment $F^{2}(0)$, and superfluid density $\rho_{s}$ versus the density $\rho$ associated with the vertical slice at $t / U_{0}=0.02$ of the phase diagram Fig. 1 The total magnetization $F_{\text {tot }}^{2}$ takes the maximum possible value $N(N+1)$.

general scaling arguments [35]. These results are in agreement with the mean field analysis [15].

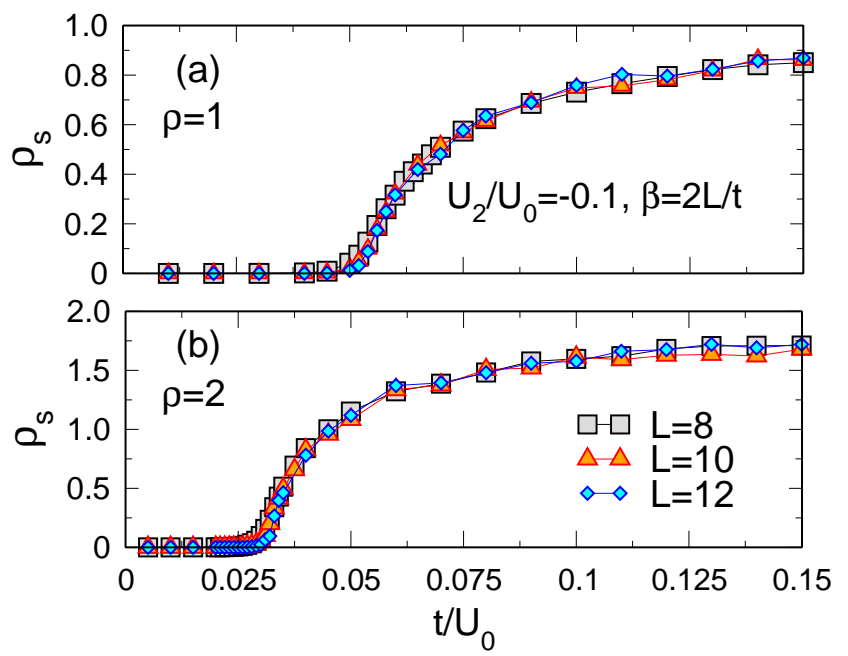

FIG. 7: (Color online) Superfluid density $\rho_{s}$ as a function of $t / U_{0}$ in the first (a) and second (b) Mott lobes. No evidence of a discontinuous jump can be seen as the system size increases; transitions are second-order.

Figure 8 shows a cut in the phase diagram as a function of $\mu$ at $t / U_{0}=0.02$ (dashed vertical line in Fig. 1) and clearly exhibits the first three incompressible Mott plateaux where the superfluid density vanishes. Here again, we do not observe discontinuities in $\rho$ or $\rho_{s}$ and conclude that the transitions are continuous. 


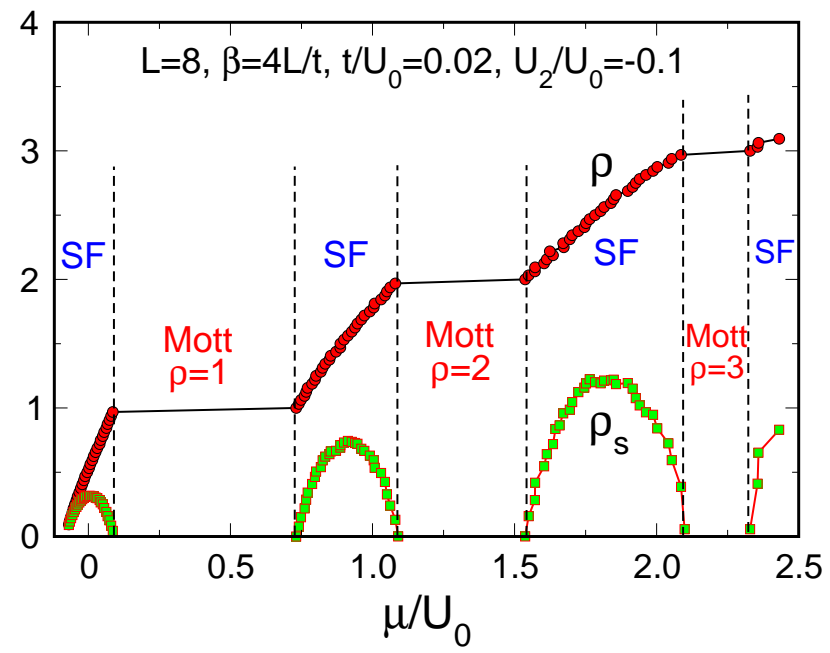

FIG. 8: (Color online) The total density, $\rho$ as a function of the chemical potential, $\mu$, showing the first three incompressible Mott phases. Also shown is the superfluid density $\rho_{\mathrm{s}}$ in the compressible phases. All the transitions are second-order.

\section{ANTIFERROMAGNETIC CASE: $U_{2}>0$}

\section{A. Phase diagram}

In the no-hopping limit, $t / U_{0} \rightarrow 0$, the system is in a MI phase with $n$ bosons per site when $\mu$ satisfies the condition $(n-1) U_{0}<\mu<n U_{0}-2 U_{2}$ for odd density and $U_{0}(n-1)-2 U_{2}<\mu<n U_{0}$ for even density. Therefore, the base of the Mott lobes for odd filling is $\Delta \mu / U_{0}=$ $1-2 U_{2} / U_{0}$, whereas it is $\Delta \mu / U_{0}=1+2 U_{2} / U_{0}$ for even filling. The even lobes grow at the expense of the odd ones, which disappear entirely for $U_{2} / U_{0}=0.5$.

The on-site energy is minimized by minimizing $F^{2}(0)$, that is by adopting one of the $F=1$ triplet states for $\rho=1$ and a unique $F=0$ singlet state for $\rho=2$, which is given at site $\mathbf{r}$ by

$$
\begin{aligned}
\left|\Phi_{\mathrm{sg}, \mathbf{r}}\right\rangle & =A_{\mathrm{sg}, \mathbf{r}}^{\dagger}|0\rangle \\
& =\frac{1}{\sqrt{6}}\left(2 a_{+\mathbf{r}}^{\dagger} a_{-\mathbf{r}}^{\dagger}-a_{0 \mathbf{r}}^{\dagger} a_{0 \mathbf{r}}^{\dagger}\right)|0\rangle
\end{aligned}
$$

This defines the singlet creation operator. Thus, in the $t / U_{0} \rightarrow 0$ limit, the MI $\rho=1$ phase is highly degenerate (as in the $U_{2}<0$ case) whereas the MI $\rho=2$ state is unique. As $t / U_{0}$ increases, quantum fluctuations cause the MI region to shrink and eventually disappear. The system is superfluid outside the MI region (Fig. 9). As in the $U_{2}<0$ case, the MF approximation [15] reproduces well the base of the Mott lobes and the tip of the $\rho=$ 2 lobe, whereas there is a significant difference for the position of the tip of the $\rho=1$ lobe. Throughout phase diagram the global magnetization is zero, $F_{\text {tot }}^{2}=0$, in the subspace $F_{\text {tot, } z}=0$. Despite this, we will see below that the two Mott lobes exhibit distinct behavior: the lifting of degeneracy in the first lobe will yield a nematic phase

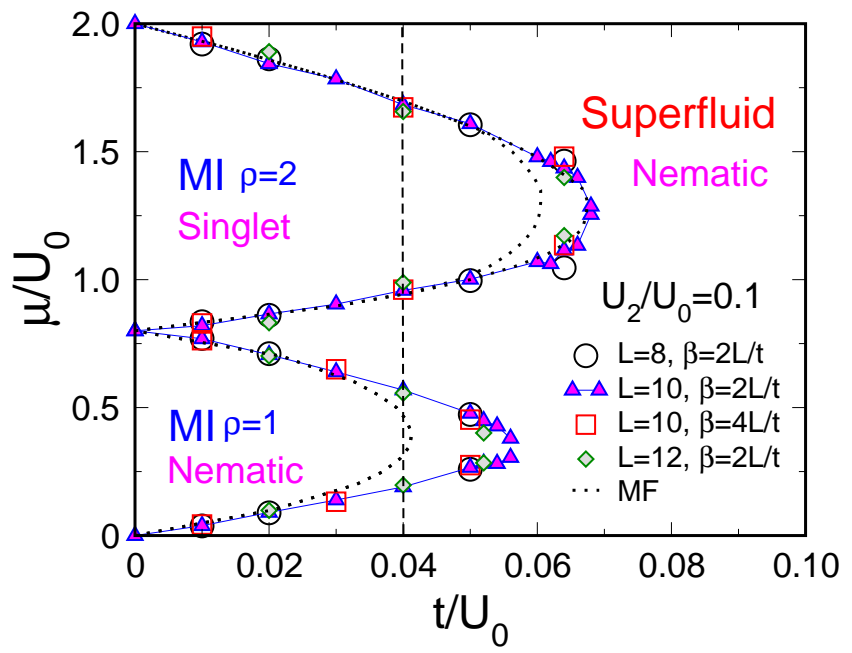

FIG. 9: (Color online) $F_{\text {tot }, z}=0$ phase diagram at $\beta=2 L / t$ or $\beta=4 L / t$ for $U_{2} / U_{0}=0.1$ and several linear system sizes $L$. In the whole phase diagram, the global magnetization is zero, $F_{\text {tot }}^{2}=0$. In the $\rho=2 \mathrm{MI}$, the on-site spin is zero, $F^{2}(0)=0$, the pair of particles forming a singlet. The $\rho=1$ MI and the superfluid phase exhibit nematic order and the system is essentially composed of spin \pm 1 particles. The MISF phase transition with $\rho=1(\rho=2)$ is second (first)-order. The dashed vertical line indicates where the cut in Fig. 17] was taken. The dotted line corresponds to the MF phase diagram obtained with a technique similar to 15], the two lines at the tip of the $\rho=2$ lobe correspond a SF-MI coexistence region.

whereas the second lobe exhibits in most cases a singlet phase, with a transition to a nematic phase under certain conditions 12.

For the $\rho=1 \mathrm{MI}$, the density distribution (Fig. 10 (a)) shows that the system is essentially composed of the $F_{z}= \pm 1$ components. These results agree with Ref. [17]. This is in contradistinction to the $U_{2}<0$ case where $\rho_{0}$ was larger than $\rho_{ \pm}$. Accordingly, in the present case, the largest Green functions will be those involving \pm 1 particles. As observed for the $U_{2}<0$ case, Fig. 10 (c) shows that $G_{a}(+-, \mathbf{R})$ quickly saturates to its maximum possible value $\rho_{-} \rho_{+}$at large distances and is larger than $G_{a}( \pm 0, \mathbf{R})$. This indicates the presence of coherent exchange moves, whereas the individual $G_{\sigma}(\mathbf{R})$ decay exponentially with distance.

In the $\rho=2 \mathrm{MI}$ phase, the system is frozen in a state with two particles per site, which form a singlet pair. In this phase, the singlet density, defined as

$$
\rho_{\mathrm{sg}}=\frac{1}{L^{2}} \sum_{r}\left\langle\hat{A}_{\mathrm{sg}, \mathbf{r}}^{\dagger} \hat{A}_{\mathrm{sg}, \mathbf{r}}\right\rangle,
$$

is approximately equal to 1 (see, for example, Fig. 20). The densities are, accordingly, $\rho_{+}=\rho_{-}=\rho_{0}=2 / 3$ as shown in Fig. 10 (b) where the density distributions are all centered around 2/3. All the Green functions decay exponentially to zero with distance (Fig. 10 (d)): the anticorrelated movements are absent in this phase because there is no degeneracy to lift when $t / U_{0} \neq 0$. 

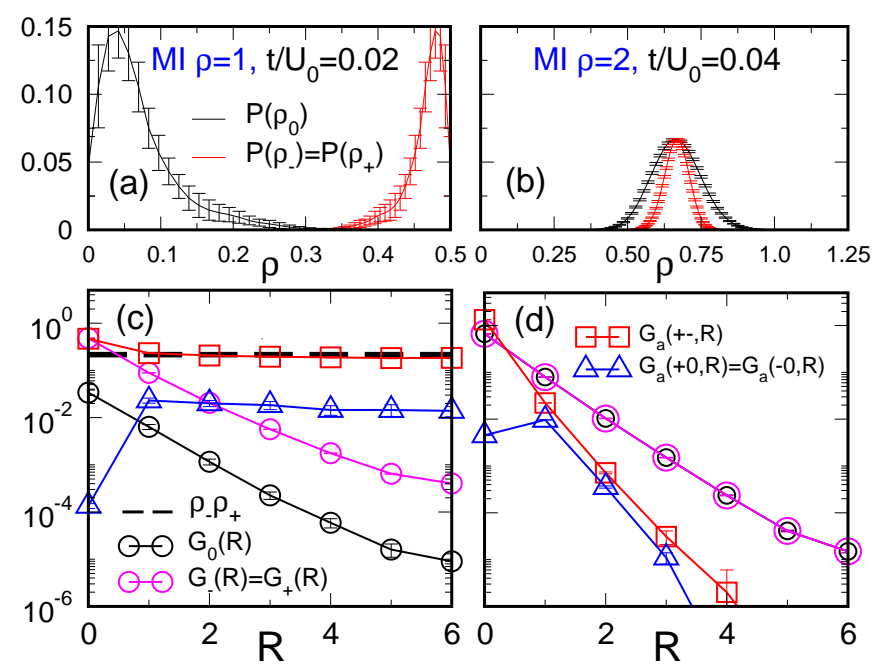

FIG. 10: (Color online) (a) and (b): Density distributions of spin- 0 and spin- \pm 1 particles in the $\rho=1,2$ MI phases and $U_{2} / U_{0}=0.1, L=12, \beta=2 L / t$. In the first lobe, there are almost only $F_{z}= \pm 1$ particles, whereas the densities of the different species are equilibrated for $\rho=2$. (c) and (d): The single-particle and the anticorrelated Green functions in the same cases. In both lobes, individual movements of particles are strongly suppressed. The first MI lobe (c) exhibits perfect anticorrelated movements of particles: $G_{a}(+-, R \rightarrow L / 2) \rightarrow$ $\rho_{-} \rho_{+}$, which is not present in the second (d).

In the superfluid phase, Fig. 111 (a) and (b) show that the peak of spin- \pm 1 distribution approaches the density $\rho / 2$, while the peak for spin- 0 approaches 0 for both densities $\rho=1,2$. Consequently, the superfluid is mostly composed of $F_{z}= \pm 1$ particles, same as in the $\rho=1 \mathrm{Mott}$ phase. This density distribution, observed in Ref. [17] for $\rho=1$, differs from the mean field one which predicts that superfluidity is carried either by the $F_{z}=0$ component or by the $F_{z}= \pm 1$ components [15]. Furthermore, Figure 11 (c) and (d) show a phase coherence both for exchange moves and for individual movements of spin- \pm 1 particles in a way similar to the $U_{2}<0$ case.

We now focus on the dependence of the global magnetization and energy on $F_{\text {tot, } z}=N_{+}-N_{-}$. According to Ref. [18], the ground state is unique, for all values of the parameters, and has a global magnetization $F_{\text {tot }}^{2}=F_{\text {tot }}\left(F_{\text {tot }}+1\right)$ with quantum number $F_{\text {tot }}=\left|F_{\text {tot }, z}\right|$ if $F_{\mathrm{tot}, z}$ is even and $F_{\mathrm{tot}}=\left|F_{\mathrm{tot}, z}\right|+1$ if $F_{\mathrm{tot}, z}$ is odd (we focus on the even $N$ case). Fig. 12 shows that the global magnetization $F_{\text {tot }}^{2}$ agrees very well with the theoretical predictions of Ref. [18] and $F_{\text {tot }}^{2}$ behaves in the same way whatever $t / U_{0}$ and $\rho$, as expected. According to Ref. [18], the ground state energy satisfies $E\left(F_{\mathrm{tot}, z}\right)<E\left(F_{\mathrm{tot}, z}+1\right)$ if $F_{\mathrm{tot}, z}$ is even and $E\left(F_{\mathrm{tot}, z}\right)=E\left(F_{\mathrm{tot}, z}+1\right)$ if $F_{\mathrm{tot}, z}$ is odd. Fig. 13 shows that this result is also verified in the whole phase diagram. This confirms that the energy is minimized when the spins are in the plane $F_{\mathrm{tot}, z}=0$ and that this constraint used in the rest of the study allows
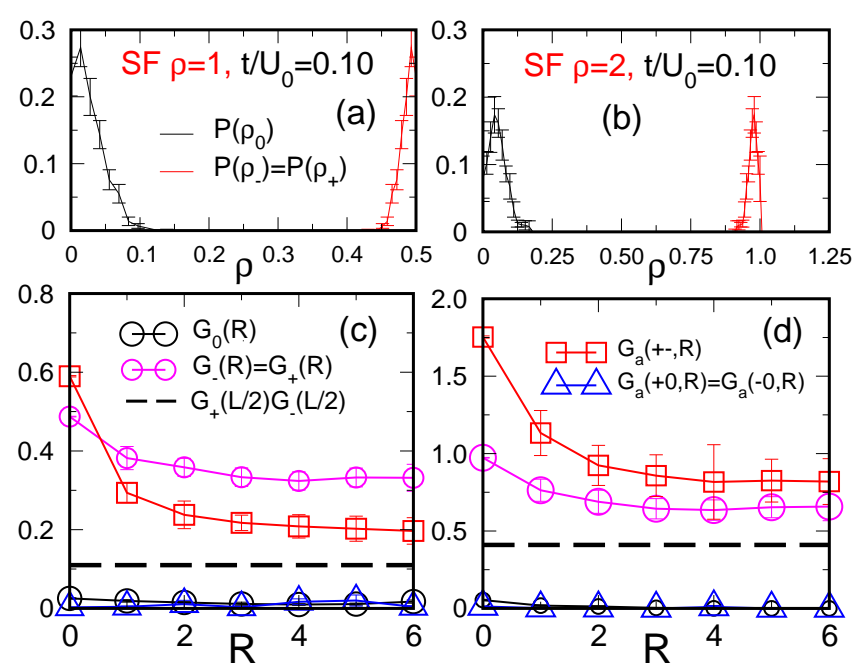

FIG. 11: (Color online) (a) and (b): Density distributions of spin- 0 and spin- \pm 1 particles in the SF phase for $\rho=1,2$ and $U_{2} / U_{0}=0.1, L=12, \beta=2 L / t$. For both $\rho=1,2$, the peak of spin- \pm 1 distribution approaches the density $\rho / 2$, while the spin-0 one approaches $0 . \quad(\mathrm{c})$ and (d): The single-particle and the anticorrelated Green functions in the SF phase in the same cases. For both $\rho=1,2$, individual movements and anticorrelated movements of spin- \pm 1 particles are present.

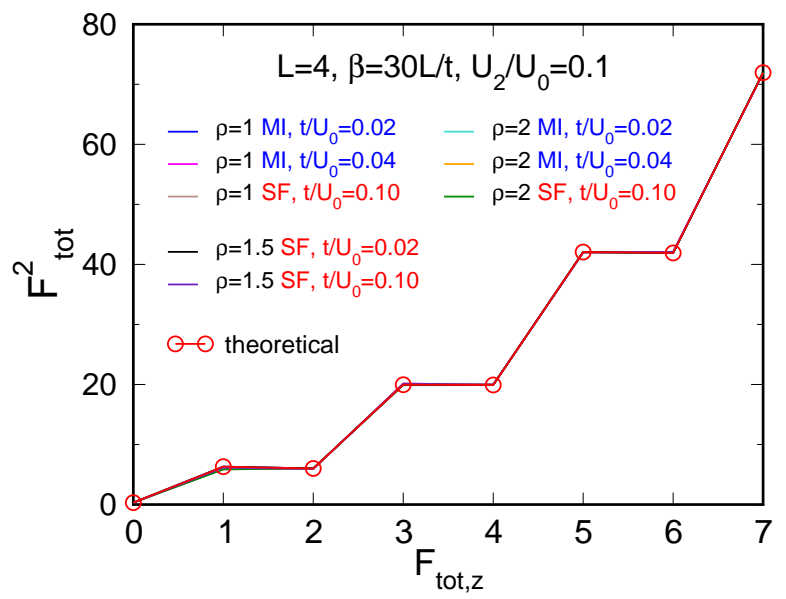

FIG. 12: (Color online) Global magnetization versus $F_{\text {tot }, z}$. The global magnetization agrees very well with the theoretical predictions of Ref. [18] and behaves in the same way whatever $t / U_{0}$ and $\rho$, as expected.

us to reach the ground state.

\section{B. Phase transitions}

An important difference between one and two dimensions is that first-order phase transitions may appear in the latter whereas they are rather rare in the former [23]. Since MFT predicts a first-order phase transition for even 

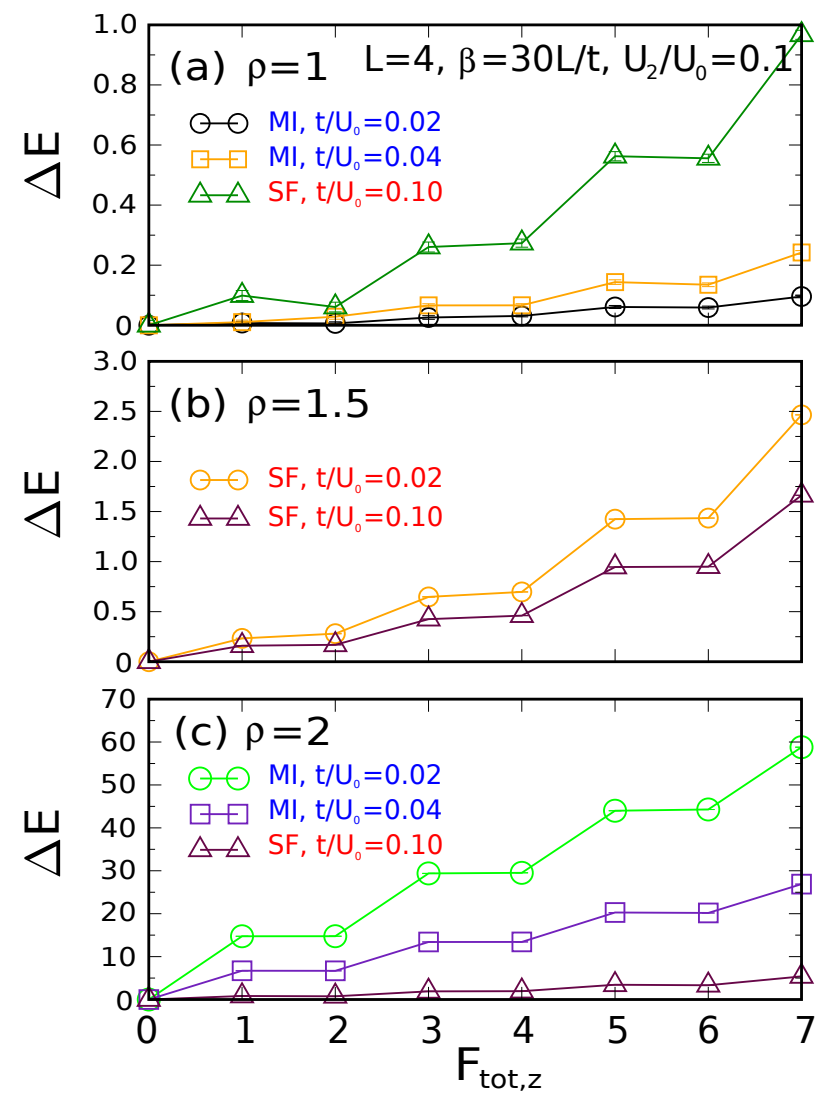

FIG. 13: (Color online) The energy difference $\Delta E=$ $E\left(F_{\text {tot }, z}\right)-E\left(F_{\text {tot }, z}=0\right)$ versus $F_{\text {tot }, z}$ in the MI and SF phases for $\rho=1$ (a), $\rho=1.5$ (b) and $\rho=2$ (c). The energy, minimum at $F_{\text {tot,z }}=0$, increases plateau by plateau with $F_{\text {tot, } z}$ as predicted in the theorem of Ref. [18].

Mott lobes 15, 16], we used QMC simulations to study this transition in detail. Figure 14 shows the evolution of the superfluid density, $\rho_{s}$, at the MI-SF transition at fixed total density while varying $t / U_{0}$. The MI-SF transition with $\rho=1$ is continuous (Fig. 14)(a)), whereas it is discontinuous with $\rho=2$ (Fig. 14 (b)): the jump in the superfluid density indicates a first-order phase transition. In Fig. 14, the finite size effects are small and the MI-SF transition with $\rho=1$ is continuous which disagrees with Ref. [17] where a first order phase transition is observed. The transition should be in the universality class of the three dimensional XY model [35].

The jump in $\rho_{s}$ at the MI-SF phase transition with $\rho=2$ is observed for different values of $U_{2} / U_{0}$. Fig. 15 shows that the transition is continuous for $U_{2} / U_{0}=0$, then it becomes discontinuous for small values, $U_{2} / U_{0}<$ 0.15 , and is again continuous for $U_{2} / U_{0} \geqslant 0.15$. The jump in the superfluid density varies continuously from zero at $U_{2} / U_{0}=0$ to a maximum for $U_{2} / U_{0} \simeq 0.05$ and then decreases back to zero as $U_{2} / U_{0}$ is increased further. This interval disagrees with the variational study [16] which predicted the first-order transition for $U_{2} / U_{0}$
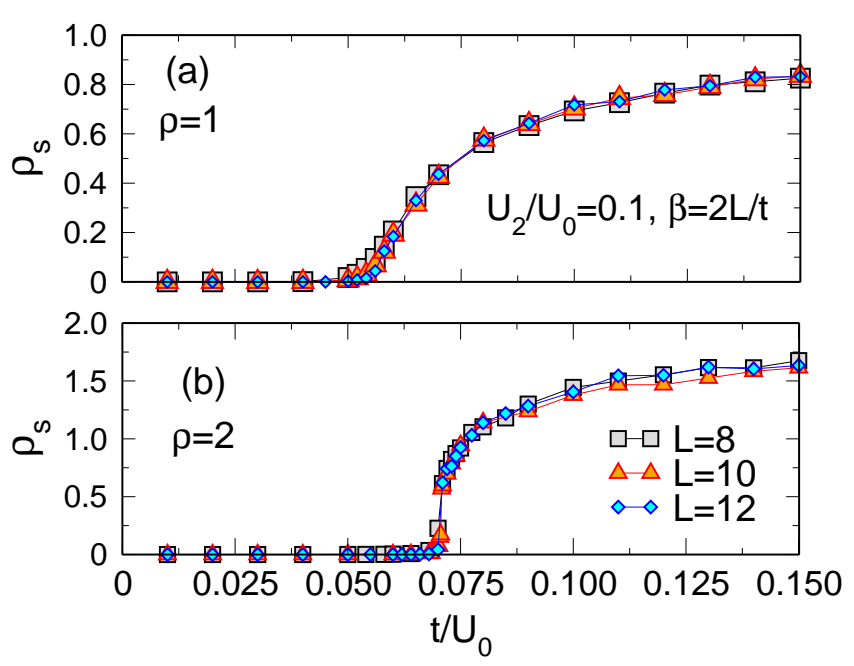

FIG. 14: (Color online) The superfluid density as a function of $t / U_{0}$ at fixed total particle density for $U_{2} / U_{0}=0.1$. The transition from the MI (small $t / U_{0}$ ) to the SF phase is signaled by $\rho_{s}$ becoming finite. For $\rho=1$ (a) the transition is continuous whereas the jump in $\rho_{s}$ marks the presence of a first-order phase transition for $\rho=2(\mathrm{~b})$.

smaller than 0.32. A similar behavior was observed for a spin $1 / 2$ model in Ref. [27].

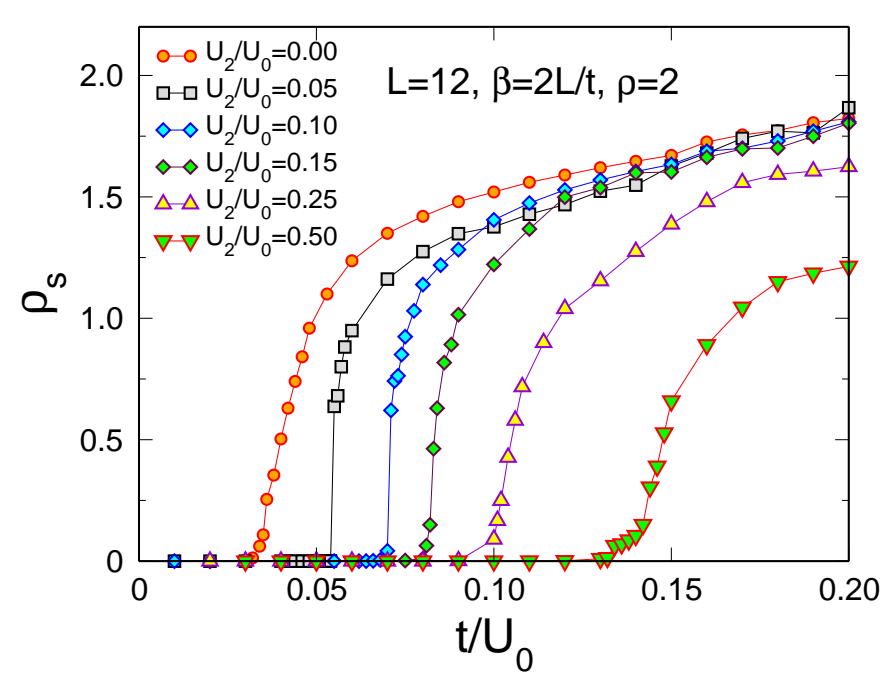

FIG. 15: (Color online) The superfluid density as a function of $t / U_{0}$ for $\rho=2$ and for several positive values of $U_{2} / U_{0}$. The jump in $\rho_{s}$ indicate the presence of a first-order transition at the tip of the $\rho=2$ Mott lobe for small but finite values of $U_{2} / U_{0}$. The jumps increase from 0 at $U_{2} / U_{0}=0$ to a maximum for $U_{2} / U_{0} \simeq 0.05$ and then decrease back to zero for $U_{2} / U_{0} \geqslant 0.15$.

To confirm the presence of first-order phase transitions near the tip of the MI lobe for even densities, we studied the behavior of $\rho$ as a function of $\mu$ as one cuts across the lobe at fixed $t / U_{0}$, using $\mathrm{QMC}$ simulations in the canonical and grand canonical ensembles. In the 


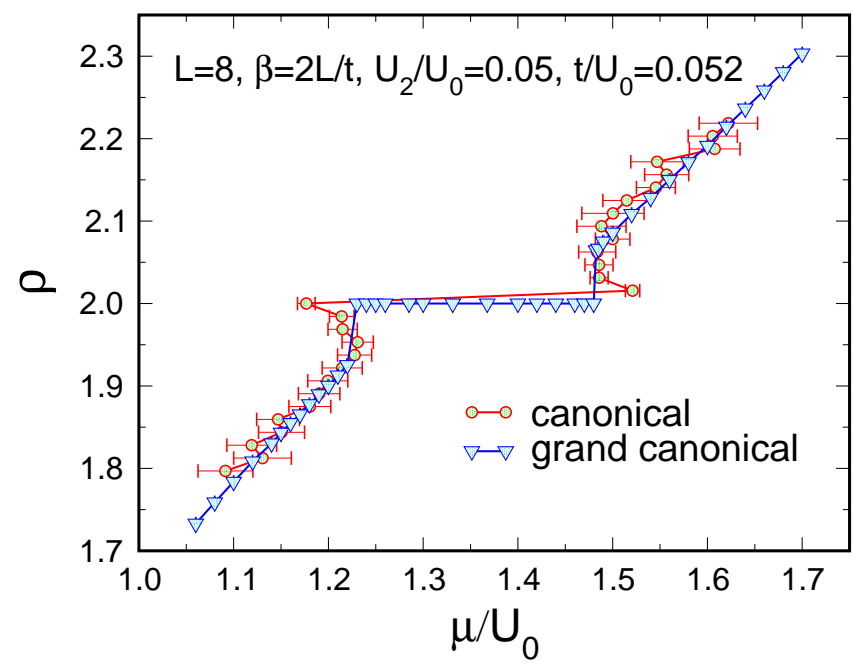

FIG. 16: (Color online) The density, $\rho$, as a function of the chemical potential, $\mu$, close to the tip of the $\rho=2$ Mott lobe with $U_{2} / U_{0}=0.05$. The canonical simulation shows a negative compressibility region, $\kappa \propto \partial \rho / \partial \mu<0$. The grand canonical simulation exhibits a corresponding jump in the density. Thus both approaches show the presence of a first-order transition.

canonical ensemble, a first-order transition is signaled by negative compressibility [36], $\kappa \propto \partial \rho / \partial \mu<0$. In the grand canonical ensemble, there will be a corresponding discontinuous jump in the $\rho$ versus $\mu$ curve. Figure 16 shows both these cases. The canonical simulations clearly show negative $\kappa$ just before and after the Mott plateau at $\rho=2$. On the other hand, the grand canonical ensemble shows discontinuous jumps in $\rho$ at the corresponding values of $\mu$. The canonical and grand canonical simulations are in quantitative agreement on the size of the unstable region, which is extremely narrow; the system is stable for densities smaller than $\rho=1.92$ or larger than $\rho=2.06$ for the chosen value of $t / U_{0}=0.052$ with $U_{2} / U_{0}=0.05$.

Figure 17 shows a vertical slice of the phase diagram for $t / U_{0}=0.04$ (Fig. 9). This slice goes through the first two incompressible Mott plateaux $(\kappa \propto \partial \rho / \partial \mu=0$ and $\rho_{s}=0$ ) but remains outside of the $\rho=3$ Mott phase. MFT predicts that the MI-SF transition near the tip of the even Mott lobes is first order [15, 16]. For $t / U_{0}=0.04, \rho_{s}$ vanishes continuously as a function of $\mu$ as the MI phase is approached and the compressibility is positive for all $\mu$, indicating that the MI-SF transitions are second order. Nevertheless, $|\kappa| \propto|\partial \rho / \partial \mu|$, increases to a huge value as the $\rho=2$ MI phase is approached. This behavior is not observed as the $\rho=1 \mathrm{MI}$ phase is approached and indicates one approaches the metastable region close to the tip of the even Mott lobes.

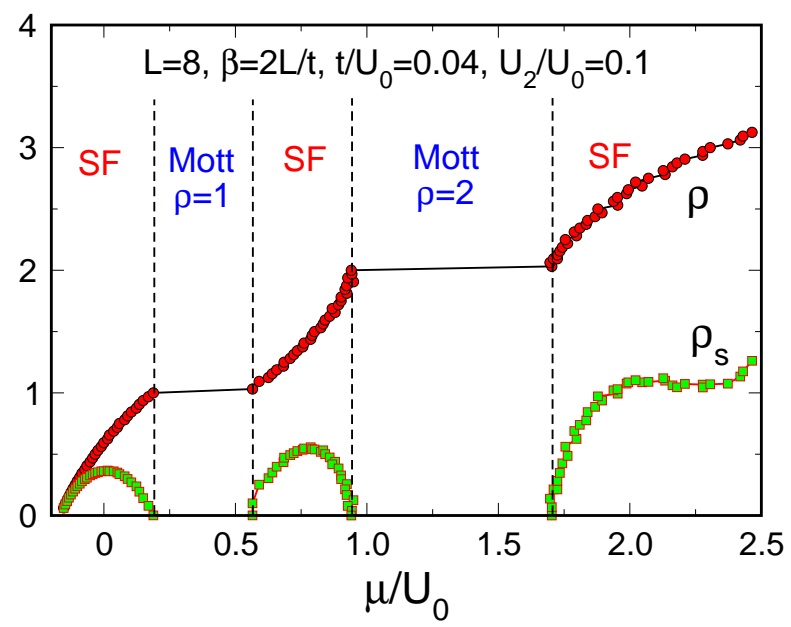

FIG. 17: (Color online) The density, $\rho$, versus chemical potential, $\mu$, exhibits the usual Mott plateaux at commensurate filling for $U_{2} / U_{0}=0.1$. Also shown is the superfluid density $\rho_{s}$ in the compressible phases. Both $\rho$ and $\rho_{s}$ are continuous as the MI phase is approached: the transitions are second order.

\section{Magnetic properties}

We now examine in detail the magnetic properties of the system and their behavior across the phase transitions. For $\rho=1$ (Fig. [18), the local moment $F^{2}(0)=2$ is constant in the Mott lobe, as one have exactly one particle per site, and slightly decreases to $F^{2}(0) \simeq 1.8$ at $t / U_{0}=0.15$ because of the fluctuations of the density in the superfluid phase. The global magnetization, equal to zero, is not affected by the transition. The nematic order parameter $Q_{z z}$ is always non zero in the Mott and superfluid phases, which indicates a spin anisotropy in these phases. At the same time, the magnetic structure factor $S(\mathbf{k})$ (Fig. 19) shows no trace of magnetic order anywhere in the phase diagram, especially no sign of antiferromagnetic spin order that would be compatible with zero total magnetization. We, therefore, conclude that there is nematic order in these two phases. Figure 18 also shows the evolution of the singlet density $\rho_{\mathrm{sg}}$ (Eq. 111) which is zero in the MI phase and remains weak in the superfluid.

Whereas the nature of magnetism does not change for the MI-SF transition with $\rho=1$, things change dramatically for $\rho=2$. In Fig. 20, the values of $t / U_{0}$ take the system from deep in the second Mott lobe $\left(\rho_{s}=0\right)$ to the superfluid phase $\left(\rho_{s} \neq 0\right)$. As shown for $\rho=1$ (Fig. 18), the global magnetization $F_{\text {tot }}^{2}$ is zero all along the transition. Deep in the MI lobe, the system has only singlets: $F^{2}(0)=0$ with $\rho_{\mathrm{sg}}=1$, while it is nematic in the superfluid phase: $F^{2}(0)$ and $Q_{z z} \neq 0$. In spite of the nematic order in the superfluid phase, pairs of singlet are still present: $\rho_{\mathrm{sg}} \neq 0$. The precision of our results does not allows us to study the coherence of singlet pairs. Thus, we cannot conclude if the su- 

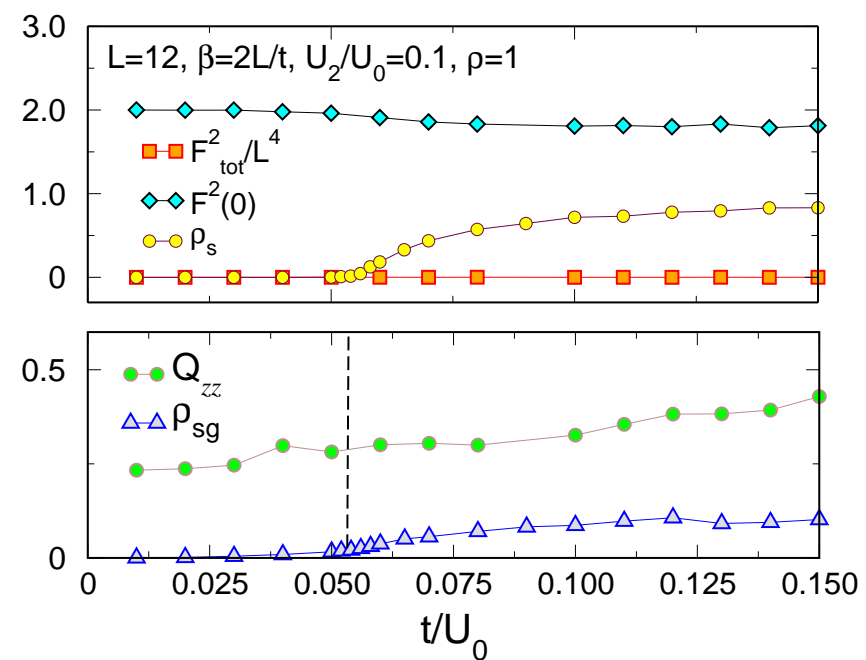

FIG. 18: (Color online) Global magnetization $F_{\text {tot }}^{2}$, local magnetic moment $F^{2}(0)$, superfluid density $\rho_{s}$, nematic order parameter $Q_{z z}$ and singlet density $\rho_{\mathrm{sg}}$ for the MI-SF transition at fixed density $\rho=1$. The system, nematic in the MI phase, remains nematic in the superfluid phase. The vertical dashed line indicates the position of the MI-SF transition.

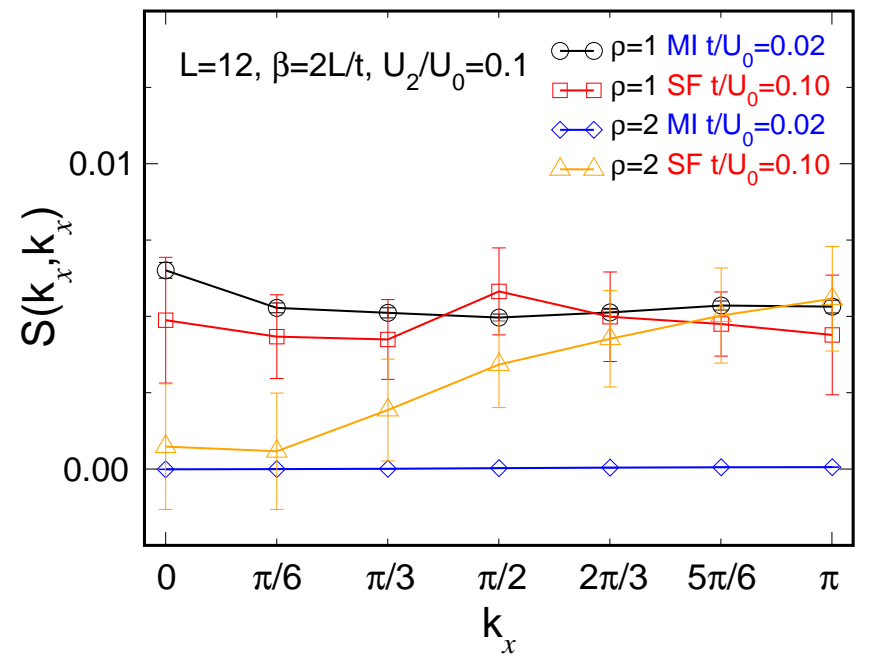

FIG. 19: (Color online) Magnetic structure factor as a function of wave vector $k_{x}$ in different phases. The magnetic structure factor always remains negligible. Especially, we do not find a peak at $\mathbf{k}=(\pi, \pi)$ that would signal an antiferromagnetic order.

perfluidity is carried by individual particles or by the singlet pairs. In the Mott lobe, close to the transition, at $t_{c} / U_{0} \simeq 0.071$, the local magnetic moment $F^{2}(0)$ increases because of the quantum fluctuations, whereas the spin remains isotropic: $Q_{z z}=0$. At the transition, simulations show discontinuous jumps in both $F^{2}(0)$ and $\rho_{s}$. The spin anisotropy, $Q_{z z} \neq 0$, grows continuously from zero in the superfluid close to the transition.

According to Refs. 12, 24, 25], for insulating states
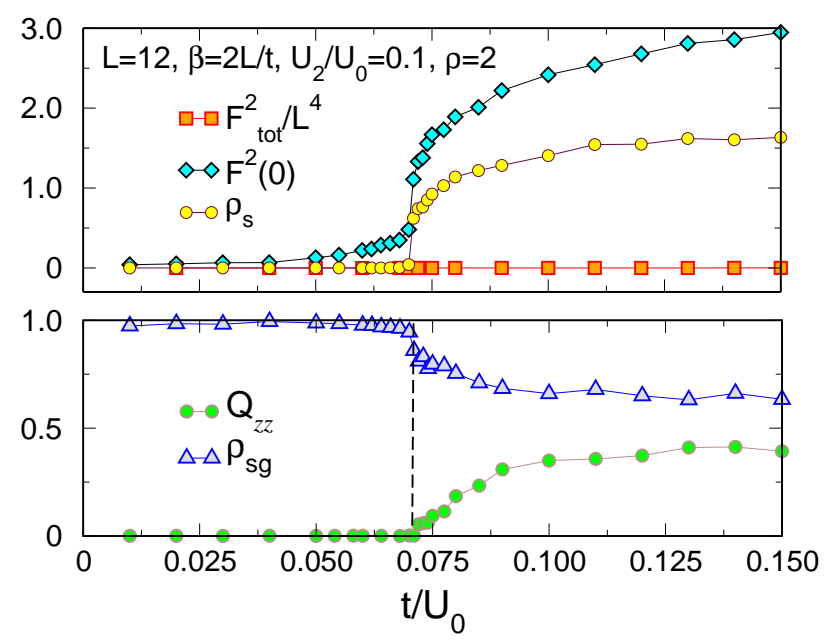

FIG. 20: (Color online) Global magnetization $F_{\text {tot }}^{2}$, local magnetic moment $F^{2}(0)$, superfluid density $\rho_{s}$, nematic order parameter $Q_{z z}$ and singlet density $\rho_{\mathrm{sg}}$ for the MI-SF transition at fixed density $\rho=2$. The system, singlet in the MI phase, becomes nematic in the superfluid phase. The vertical dashed line indicates the position of the MI-SF transition.

with an even number of particles per site, there is always a spin singlet phase, but there may also be a first order transition between a singlet and a nematic phase inside the $\rho=2$ Mott phase for small values of $U_{2} / U_{0}<0.025$ in two dimensions. In Fig. 21 we elucidate this question by using smaller values of $U_{2} / U_{0}: 0.01$ and 0.005 . As observed for $U_{2} / U_{0}=0.10$, the system is singlet deep in the Mott lobe and nematic in the superfluid phase. Figure 21 (top) shows that the SF-MI transition, $\rho_{s} \rightarrow 0$, occurs at a larger $t / U_{0}$ than the transition to nematic or$\operatorname{der} Q_{z z} \rightarrow 0$ for $U_{2} / U_{0}=0.01$. Thus the singlet-nematic transition seems to take place inside the MI phase, but quite close to the MI-SF transition. More interestingly, we see that the position of the singlet-nematic transition is shifted to the left with increasing sizes whereas the position of the MI-SF transition is almost unchanged, which gives a clearer separation of the two transition points for larger sizes. For example, for $L=12$, the MI-SF transition happens for $t / U_{0} \simeq 0.037$, whereas the nematic transition happens for $t / U_{0} \simeq 0.031$. This effect is more pronounced in the second case $U_{2} / U_{0}=0.005$ (Fig. 21 (bottom)) where the transitions take place at $t / U_{0} \simeq 0.037$ and $t / U_{0} \simeq 0.025$ respectively and where the nematic region in the Mott phase is much wider. In both these cases, it is difficult to conclude if there is a first or a second order MI-SF transition. We observe a seemingly continuous transition but we are close to $U_{2} / U_{0}=0$ where the transition is continuous. If there is a discontinuity, we would except the jump in $\rho_{s}$ to be very small and, hence, very difficult to observe. In the $U_{2} / U_{0}=0.01$ case, there is also no evidence of a first-order transition for the singlet-nematic transition, as $F^{2}(0)$ and $Q_{z z}$ evolve in a seemingly continuous way 
with our precision (Fig. 21](top)). However, a discontinuity is observed in these quantities (Fig. 21 (bottom)) for the largest size, $L=12$, for $U_{2} / U_{0}=0.005$; this signals a discontinuous transition. A rigorous investigation with larger sizes $L>12$, currently inaccessible with QMC, would be required to specify the exact point where the transition occurs and to see if this discontinuous transition is also present in the $U_{2} / U_{0}=0.01$ case. We have also studied the $U_{2} / U_{0}=0.05$ case but it was impossible to distinguish the MI-SF phase transition from the singlet-nematic one, which then appears similar to the $U_{2} / U_{0}=0.10$ case.

Figure 22 shows the local magnetic moment, $F^{2}(0)$, the singlet density, $\rho_{\mathrm{sg}}$, the superfluid density, $\rho_{s}$, the nematic order parameter, $Q_{z z}^{\prime}$, and the global magnetization, $F_{\text {tot }}^{2}$, versus the density taken from Fig. 17. All along this slice, the global magnetization $F_{\text {tot }}^{2}$ is zero, as expected. As the density is increased, we see that the number of singlet increases regularly for $\rho \geq 1$ as expected. The behavior of the local magnetic moment can be understood in terms of a minimization process. For an even number of particles on a site, the minimum value of the local moment is zero whereas it is $F^{2}(0)=2$ for an odd number of particles. We then observe this alternation of increases of the local moment when the number of sites with an odd number of particles is increasing $(\rho<1$ or $2<\rho<3$ ) and of decreases when the number of sites with an even number is increasing $(1<\rho<2)$. In this case, we measured $Q_{z z}^{\prime}$ because $Q_{z z}$ is ill behaved for $\rho<1$, as explained in section $[1$ We find that the spin anisotropy and nematic order is present in the whole superfluid phase, whatever the density. It is also present, as seen before, in odd density Mott phases and essentially absent from even density Mott phases.

\section{CONCLUSION}

We studied spin-1 bosons with interactions on a lattice. The interactions are among the simplest possible for such a system: an on-site repulsion independent of spin and an on-site ferromagnetic or antiferromagnetic coupling between spins on the same site.

We have shown that, for both ferromagnetic $\left(U_{2}<\right.$ $0)$ and antiferromagnetic $\left(U_{2}>0\right)$ interactions, our QMC results are in good agreement with the theorem of Ref. [18].

For $U_{2}<0$ we showed that all MI-SF transitions are continuous and that the whole phase diagram is ferromagnetic. This is very similar to what was observed in the one dimensional case [23], although the nature of the MI-SF transitions with fixed integer density differs from the $1 \mathrm{D}$ case. The ferromagnetism of the whole phase diagram was demonstrated by Katsura and Tasaki and we verified numerically their theorem. We also determined the extent of the different Mott and superfluid phases and showed that the magnetic behavior is indeed not affected by the different natures (superfluid or solid) of theses
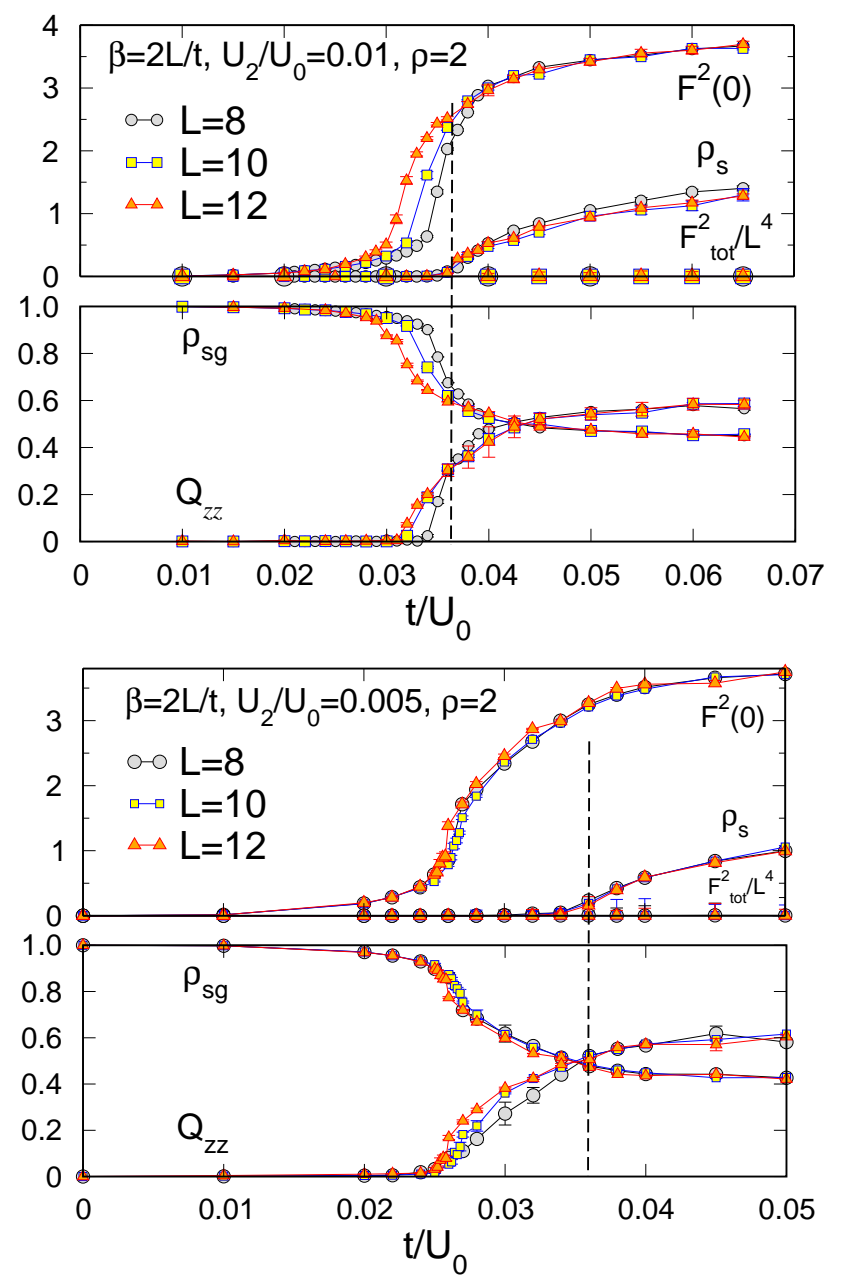

FIG. 21: (Color online) Global magnetization $F_{\text {tot }}^{2}$, local magnetic moment $F^{2}(0)$, superfluid density $\rho_{s}$, nematic order parameter, $Q_{z z}$, and singlet density $\rho_{\mathrm{sg}}$ for the MI-SF transition at fixed density $\rho=2$ for several system sizes $L$ and for two values of $U_{2} / U_{0}: U_{2} / U_{0}=0.01$ (top) and $U_{2} / U_{0}=0.005$ (bottom). Deep in the Mott lobe, the system is singlet whereas the system is nematic in the superfluid phase. The singletnematic transition seems to appear in the MI lobe, close to the transition, as $L$ is increased. In the case where $U_{2} / U_{0}=0.005$, the simulation on the largest $L=12$ size shows a discontinuity in the evolution of the physical quantities, thus indicating a possible first order transition between a singlet and a nematic Mott insulator phase. The vertical dashed lines indicate the positions of the MI-SF transitions.

phases. This is rather surprising as our model does not include explicit coupling between spins on different sites. These couplings arise due to the movement of the particles, and these movements are quite different in solid and superfluid phases.

For $U_{2}>0$, the situation is more interesting as Katsura and Tasaki's theorem only predicts the global value of the magnetization $F_{\text {tot }}^{2}$ to be zero in the simple case where $F_{\text {tot, } z}=0$. This value is compatible with several magnetic orders, namely an antiferromagnetic order or a 


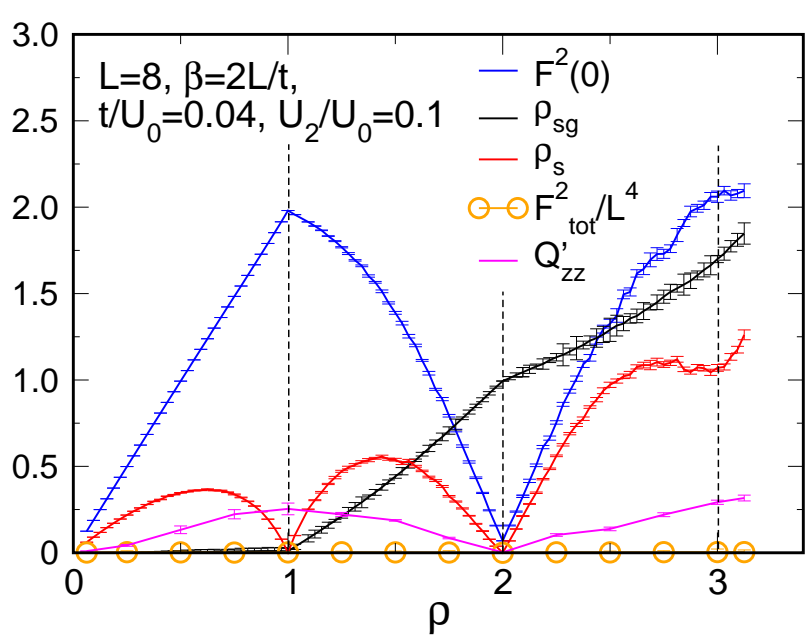

FIG. 22: (Color online) Local magnetic moment $F^{2}(0)$, singlet density, $\rho_{\mathrm{sg}}$, superfluid density, $\rho_{s}$, global magnetization $F_{\text {tot }}^{2}$, and nematic order parameter $Q_{z z}^{\prime}$ versus the density $\rho$ associated with the vertical slice at $t / U_{0}=0.04$ of the phase diagram Fig. 9

nematic order, and the theorem does not specify which order, if any, will emerge in the ground state. Analysing the magnetic structure factor, we excluded the possibility of antiferromagnetic order. The first Mott lobe was expected to be nematic [12, 17] via a mapping on a spin1 Heisenberg model and, indeed, we found that the nematic order parameter $Q_{z z}$ was non zero in this phase. More surprinsingly, nematic order is also present in the superfluid phase. Furthermore, the density distribution differs from that expected from MFT [15] which predicts another possible ground state where the spin-0 population dominates. In the second Mott lobe, we observe the formation of singlet order for $U_{2} / U_{0}=0.1$, and a singletnematic transition inside the Mott lobe, close to the tip of the lobe with $U_{2} / U_{0}=0.01$ and $U_{2} / U_{0}=0.005$ as expected [12]. We observed such a transition on the sizes we could study, and were able to find evidence of a first order transition for the $U_{2} / U_{0}=0.005$ case (although we did not observe such evidence for $U_{2} / U_{0}=0.01$ ) as predicted with MFT in 12, 24, 25]. Finally, we demonstrate that the MI-SF transition in the $\rho=1$ lobe is continuous, independently of $U_{2}$, whereas the transition in the $\rho=2$ lobe is discontinuous (first-order) for $0.05<U_{2} / U_{0}<0.15$ and continuous for larger values.

\section{Acknowledgments}

This work was supported by: the CNRS-UC Davis EPOCAL LIA joint research grant; by NSF grant OISE0952300; and ARO Award W911NF0710576 with funds from the DARPA OLE Program.
[1] I. Bloch, J. Dalibard, and W. Zwerger, Rev. Mod. Phys. 80, 885 (2008).

[2] M. Greiner, O. Mandel, T. Esslinger, T.W. Hänsch, and I. Bloch, Nature 415, 39 (2002).

[3] U. Schneider, L. Hackermüller, S. Will, Th. Best, I. Bloch, T. A. Costi, R. W. Helmes, D. Rasch, and A. Rosch, Science 322, 5907 (2008).

[4] R. Jördens, N. Strohmaier, K. Günter, H. Moritz, and T. Esslinger, Nature 455, 204 (2008).

[5] D. M. Stamper-Kurn, M. R. Andrews, A. P. Chikkatur, S. Inouye, H.-J. Miesner, J. Stenger, and W. Ketterle, Phys. Rev. Lett. 80, 2027 (1998).

[6] M. Vengalattore, S. R. Leslie, J. Guzman, and D. M. Stamper-Kurn, Phys. Rev. Lett. 100, 170403 (2008).

[7] M. Vengalattore, J. Guzman, S. R. Leslie, F. Serwane, and D. M. Stamper-Kurn, Phys. Rev. A 81, 053612 (2010).

[8] T.-L. Ho, Phys. Rev. Lett. 81, 742 (1998).

[9] T. Ohmi and K. Machida, J. Phys. Soc. Jpn. 67, 1822 (1998).

[10] M. Theis, G. Thalhammer, K. Winkler, M. Hellwig, G. Ruff, R. Grimm, and J. H. Denschlag, Phys. Rev. Lett. 93, 123001 (2004); G. Thalhammer, M. Theis, K. Winkler, R. Grimm, and J. H. Denschlag, Phys. Rev. A 71, 033403 (2005).

[11] D. M. Stamper-Kurn and W. Ketterle, in Coherent Atomic Matter Waves, edited by R. Kaiser, C. Westbrook, and F. David (Springer, Berlin, 2001), p. 137.
[12] A. Imambekov, M. Lukin, and E. Demler, Phys. Rev. A 68, 063602 (2003) and Phys. Rev. Lett. 93, 120405 (2004).

[13] N. Kawashima, Prog. Theor. Phys. Suppl. 145, 138 (2002).

[14] S. Tsuchiya, S. Kurihara, and T. Kimura, Phys. Rev. A 70, 043628 (2004).

[15] R.V. Pai, K. Sheshadri, and R. Pandit, Phys. Rev. B 77, 014503 (2008).

[16] T. Kimura, S. Tsuchiya, and S. Kurihara, Phys. Rev. Lett. 94, 110403 (2005).

[17] Y. Toga, H. Tsuchiura, M. Yamashita, K. Inaba, and H. Yokoyama, J. Phys. Soc. Jpn. 81, 063001 (2012).

[18] H. Katsura and H. Tasaki, Phys. Rev. Lett. 110, 130405 (2013).

[19] T. Kimura, Phys. Rev. A 87, 043624 (2013).

[20] M. Rizzi, D. Rossini, G. De Chiara, S. Montangero, and R. Fazio, Phys. Rev. Lett. 95, 240404 (2005).

[21] S. Bergkvist, I.P. McCulloch, and A. Rosengren, Phys. Rev. A 74, 053419 (2006).

[22] V. Apaja and O. F. Syljuåsen, Phys. Rev. A 74, 035601 (2006).

[23] G. G. Batrouni, V. G. Rousseau, and R. T. Scalettar, Phys. Rev. Lett. 102, 140402 (2009).

[24] E. Demler and F. Zhou, Phys. Rev. Lett. 88, 163001 (2002).

[25] M. Snoek and F. Zhou, Phys. Rev. B 69, 094410 (2004).

[26] L. de Forges de Parny, M. Traynard, F. Hébert, V. G. 
Rousseau, R. T. Scalettar, and G. G. Batrouni, Phys. Rev. A 82, 063602 (2010).

[27] L. de Forges de Parny, F. Hébert, V. G. Rousseau, R .T. Scalettar, and G. G. Batrouni, Phys. Rev. B 84, 064529 (2011).

[28] K. V. Krutitsky and R.Graham, Phys. Rev. A 70, 063610 (2004).

[29] K. V. Krutitsky, M.Timmer, and R.Graham, Phys. Rev. A 71, 033623 (2005).

[30] V. G. Rousseau, Phys. Rev. E 77, 056705 (2008).

[31] V. G. Rousseau, Phys. Rev. E 78, 056707 (2008).
[32] D. M. Ceperley and E. L. Pollock, Phys. Rev. B 39, 2084 (1989).

[33] A. B. Kuklov and B. V. Svistunov, Phys. Rev. Lett. 90, 100401 (2003).

[34] B. Capogrosso-Sansone, S. G. Söyler, N. V. Prokofév and B. V. Svistunov, Phys. Rev. A 81, 053622 (2010).

[35] M. P. A. Fisher, P. B. Weichman, G. Grinstein, and D. S. Fisher, Phys. Rev. B 40, 546 (1989).

[36] G. G. Batrouni and R. T. Scalettar, Phys. Rev. Lett. 84, 1599 (2000). 